\section{Recomendaciones de la OMS para gobiernos y consumidores acerca del uso de los tratamientos tradicionales $^{1}$}

Palabras clave: medicina tradicional, naturopatía, medicina herbaria, Organización Mundial de la Salud.

\footnotetext{
Basado en World Health Organization, Guidelines on Developing Consumer Information on Proper Use of Traditional, Complementary and Alternative Medicine, enero de 2004. El documento se puede consultar en el sitio web de la Organización Mundial de la Salud: http:// www.who.int/en/
}

En junio de este año la Organización Mundial de la Salud (OMS) emitió una serie de recomendaciones para fomentar el uso adecuado de los llamados tratamientos tradicionales, complementarios o "alternativos". Se calcula que alrededor de $80 \%$ de las poblaciones de países en desarrollo dependen principalmente de los remedios caseros y herbarios y de otros tipos de tratamientos tradicionales, como la acupuntura, para calmar sus dolencias corrientes. Estos tratamientos también están gozando de una popularidad inusitada en sociedades prósperas. Entre otras cosas, son baratos, abundantes, fáciles de elaborar y conseguir, y más "sanos", a ojos del público, que los fármacos sintéticos. Se suma a estos factores el hecho de que hoy en día los habitantes de países desarrollados cuidan de su salud más activamente que en épocas pasadas y tienen a su alcance opciones terapéuticas más diversas, entre ellas las menos convencionales. Según una gran encuesta multinacional que realizó la OMS, la mayoría de los medicamentos herbarios o naturales se obtienen sin receta en casi todas partes y los habitantes los usan libremente para tratarse a sí mismos o a sus parientes y amigos. No cabe duda de que los tratamientos tradicionales, bien aplicados, pueden representar un componente valioso de los sistemas de salud de muchos países.

\section{Riesgos y beneficios de los tratamientos alternativos}

La mayor parte de los remedios naturales que se venden sin receta médica y los de preparación doméstica son inocuos y pueden ser beneficiosos cuando se utilizan correctamente; cuando no, pueden ser dañinos y peligrosos, como confirman informes recientes de reacciones adversas y otros efectos. Al no estar sujetos a un control de calidad oficial en muchos países, su calidad y autenticidad varían ostensiblemente. Muchos carecen de eficacia comprobada y pueden provocar reacciones inesperadas. En la China, por ejemplo, se documentaron 9854 casos de reacciones adversas a tratamientos de este tipo en 2002, más del doble de las notificadas en 1990. A ello se suma que las personas a menudo los utilizan sin la supervisión de un médico y sin conocer sus propiedades o dosis adecuadas ni para qué sirven. Y como cabe esperar, quienes practican la acupuntura y otros tratamientos mecánicos o manuales no siempre tie- 
nen la experiencia ni los conocimientos necesarios para no lesionar al paciente.

Muchos tratamientos tradicionales o alternativos tienen una eficacia indiscutible que ha sido demostrada en ensayos clínicos; tal es el caso de la acupuntura y de remedios como Hypericum perforatum ("hierba de San Juan") y Serenoa repens, que se usan para el tratamiento de la depresión leve y de la hiperplasia prostática benigna, respectivamente. En el caso de la acupuntura, los Institutos Nacionales de Salud de los Estados Unidos de América han afirmado en una declaración consensuada que esta técnica se acompaña de una incidencia de efectos nocivos sumamente baja y hasta menor que la observada con técnicas terapéuticas convencionales. No obstante, en la mayoría de los casos las "pruebas" de eficacia son empíricas; provienen de farmacopeas, fuentes apócrifas, y de las experiencias y conocimientos acumulados por la humanidad desde tiempos inmemoriales. Falta investigar mediante ensayos clínicos la verdadera eficacia de muchos de estos remedios. Y aun cuando muchos productos y técnicas sí alivian las dolencias, también se han observado problemas de salud como resultado de su uso. Ha habido casos de sobredosis involuntarias, reacciones nocivas, interacciones medicamentosas, consumo de productos falsificados, equivocados o adulterados y lesiones graves a causa de manipulaciones físicas realizadas por terapeutas que carecen de experiencia o que no tienen en cuenta el estado físico subyacente del cliente. Algunos productos están contaminados con metales pesados, plaguicidas, toxinas microbianas y hasta sustancias farmacéuticas en cantidades nocivas. La situación coloca al público en peligro de sufrir algún tipo de daño cuando faltan mecanismos que garanticen la administración y el uso adecuados de estos tratamientos. De ahí la importancia de proporcionar información clara, concisa y de fácil acceso para orientar a las personas en su elección de tratamientos alternativos y ayudarlas a conocer mejor sus riesgos y beneficios.

\section{La importancia de la información pública}

La Asamblea Mundial de la Salud adoptó en 2003 una resolución donde se instaba a los Estados Miembros de la OMS a proporcionar a los consumidores y proveedores de atención información fiable sobre los tratamientos tradicionales, y en consonancia con ello la OMS formuló el documento titulado WHO Guidelines: Developing Consumer Information on Proper Use of Traditional, Complementary and Alternative Medicine. Este extenso documento con pautas, elaborado en colaboración con la Universidad de Milán, Italia, con el apoyo técnico y económico del Gobierno Regional de Lombardía y con fondos aportados por el Gobierno de Suecia, contiene la información necesaria para ayudar a los gobiernos, organizaciones no gubernamentales y otras partes interesadas a crear y diseminar por los medios de comunicación en masa información pública adecuada acerca de las prácticas terapéuticas tradicionales. En ella se recomiendan medidas y procesos para fomentar el uso adecuado de los productos y tratamientos tradicionales en el contexto de los sistemas de salud existentes y se provee una lista de pautas y preguntas muy sencillas para que el consumidor sepa en qué fijarse a la hora de elegir un tratamiento tradicional, complementario o "alternativo".

Las recomendaciones toman en cuenta la necesidad de adaptar la información elaborada a distintos contextos sociales, culturales y religiosos. En algunos países donde el nivel de escolaridad es bajo, por ejemplo, hay que usar más ilustraciones y un lenguaje más sencillo que en países donde la escolaridad es mayor. Para que surtan los efectos deseados, las recomendaciones deberán verse respaldadas por leyes y reglamentos oficiales que garanticen la calidad de los remedios herbarios y la buena formación y certificación de quienes se dedican a la práctica profesional de las técnicas terapéuticas alternativas. Para facilitar la investigación es necesario reconocer legalmente el potencial terapéutico de estas, obtener financiamiento, idear métodos de investigación idóneos e implantar sistemas para proteger la propiedad intelectual. Finalmente, son muchas las partes que deberán participar en el esfuerzo por lograr el uso sin riesgo de los tratamientos alternativos: gobiernos, consumidores, organizaciones gremiales y no gubernamentales, investigadores y autoridades sanitarias. A continuación se enumeran algunos de los aspectos sobresalientes de las recomendaciones dirigidas a los gobiernos y al consumidor.

\section{Estructuras y procesos para facilitar el uso adecuado de los tratamientos tradicionales}

Dentro del contexto de los sistemas de salud es imprescindible contar con ciertas estructuras y procesos clave que permitan fomentar la educación, la investigación y la provisión de información adecuada en torno a los tratamientos tradicionales. Algunas medidas deberán observarse, entre ellas:

- la creación de estándares y de sistemas reguladores para el control de la calidad

- la elaboración de pautas terapéuticas fiables basadas en los resultados de ensayos clínicos y otros estudios científicos 
- el adiestramiento estandarizado y la acreditación de los profesionales calificados en la aplicación de técnicas terapéuticas tradicionales con miras a mejorar su credibilidad y la confianza del público

- el fomento de una colaboración más estrecha entre los profesionales de la medicina tradicional y los de la medicina convencional a fin de mejorar los resultados del tratamiento y lograr la reforma del sector sanitario

- el mejoramiento de la comunicación entre los consumidores y los profesionales de la medicina tradicional y de la medicina convencional

- la creación de organizaciones locales de profesionales dedicados a la medicina tradicional como primer paso hacia la formación de organizaciones nacionales, todo con el propósito de facilitar la adopción de mecanismos de control gremial.

\section{Políticas que los gobiernos podrían adoptar}

Para fomentar el uso adecuado de los tratamientos tradicionales, hay una serie de medidas que los gobiernos deberían emprender.

- cerciorarse de que los consumidores tengan a su alcance suficiente información sobre la eficacia e inocuidad de los productos tradicionales y sus contraindicaciones

- establecer y dar a conocer los canales apropiados para que los consumidores puedan notificar reacciones adversas

- organizar campañas de comunicación destinadas a dotar a los consumidores de la capacidad para saber cuál es la calidad de los servicios que reciben

- asegurarse de que los profesionales dedicados al ejercicio de la medicina tradicional tengan la capacitación y certificación debidas

- fomentar la interacción entre los profesionales dedicados a la medicina tradicional y los que cultivan la medicina convencional

- proveer seguros que cubran los tratamientos y productos tradicionales cuya eficacia e inocuidad se hayan demostrado.

\section{Preguntas que debe plantearse el consumidor}

En el momento de querer usar un producto herbario o someterse a un tratamiento basado en técnicas tradicionales, el consumidor debe plantearse las siguientes preguntas básicas:

- ¿Es apropiado el tratamiento para aliviar mi afección?
- ¿Puede este tratamiento prevenir, aliviar o curar los síntomas que tengo o de algún otro modo mejorar mi salud y bienestar?

- ¿Administra el tratamiento o el producto herbario una persona calificada (de preferencia registrada y certificada) o un profesional de la salud que posea la formación, la experiencia y los conocimientos adecuados?

- ¿Está garantizada la calidad de los productos o materiales medicinales herbarios y en qué casos está contraindicado su uso o es preciso tomar precauciones?

- ¿Pueden conseguirse estos tratamientos o productos medicinales herbarios a un precio razonable?

\section{Conclusiones}

Los remedios caseros y los productos naturales pueden ser beneficiosos cuando se usan con cautela y con un conocimiento básico de sus propiedades, efectos y riesgos. En muchas sociedades en desarrollo representan el recurso terapéutico más usado por los habitantes, y su popularidad en sociedades desarrolladas es cada vez mayor debido a su accesibilidad, bajo costo y "pureza". No obstante, estos remedios, así como la acupuntura y otras prácticas tradicionales a base de maniobras físicas, pueden provocar reacciones adversas y perjudicar la salud. Por consiguiente, es necesario que los gobiernos nacionales y otras entidades elaboren información adecuada para el público y adopten medidas que fomenten el uso adecuado de estos tratamientos dentro de los sistemas de salud de los países. Ello implica, entre otras cosas, facilitar los vínculos profesionales entre los terapeutas de la medicina tradicional y los que se dedican a la medicina convencional, así como adoptar leyes que regulen la calidad de los tratamientos y la formación y acreditación de quienes los administran. Se espera que con el esfuerzo concertado de las diversas partes - los gobiernos, sociedades de profesionales, agencias no gubernamentales y los propios consumidores- se logre crear las condiciones propicias para aprovechar el potencial que ofrecen los tratamientos tradicionales para mejorar la salud de los pueblos.

\section{SYNOPSIS}

WHO guidelines for governments and consumers regarding the use of alternative therapies

Herbal remedies and other alternative treatments, such as acupuncture and homeopathy, are widely used in traditional 
societies and are becoming immensely popular in developed countries as well. Most are cheap, readily available, easy to use, and are perceived to be "healthier" than pharmaceutical substances. However, users need to be better informed as to the actual benefits and properties of these forms of therapy and their potential hazards. Improper use puts consumers at risk for potentially serious side effects as a result of allergy, drug interactions, contamination of the products with pesticides, heavy metals, and other substances, or trauma inflicted by inexperienced or poorly trained practitioners. Some traditional remedies can cause undesirable effects, such as excessive bleeding. Thus, it is essential that users have access to information that is reliable, clear, and easily available.

In June of this year the World Health Organization released a new set of guidelines to help national health author- ities develop and distribute this type of information. The Guidelines on Developing Consumer Information on Proper Use of Traditional, Complementary and Alternative Medicine, which were developed with the financial and technical support of the Government of Lombardy, Italy, and the Government of Sweden, are part of a collaborative project that seeks to promote the proper use of traditional, complementary, and alternative therapies by consumers. In addition to offering advice for government officials on how to prepare reliable consumer information, the guidelines contain a series of simple questions that can guide users through the process of deciding on the use of such therapies. The guidelines are published in English at the World Health Organization's website: http://www.who.int/en/

\begin{tabular}{|c|}
\hline $\begin{array}{c}\text { V Curso de Experto Universitario y Diploma de Especialización } \\
\text { en Epidemiología y Nuevas Tecnologías Aplicadas }\end{array}$ \\
\hline $\begin{array}{l}\text { Fecha: noviembre de } 2004 \text { a septiembre de } 2005 \\
\text { Lugar: Madrid, España }\end{array}$ \\
\hline $\begin{array}{l}\text { El Centro Nacional de Epidemiología y la Escuela Nacional de Sanidad, del Insti- } \\
\text { tuto de Salud Carlos III en Madrid, España, se complacen en anunciar la celebración de } \\
\text { este curso de capacitación cuya duración es de un año académico. El curso busca en- } \\
\text { señarles a los participantes a: a) describir, analizar e interpretar problemas epidemioló- } \\
\text { gicos en el ámbito de la salud pública; b) tener nociones de estadística clásica, estadís- } \\
\text { tica bayesiana y diseño y desarrollo de modelos de enfermedad dinámico-matemáticos; } \\
\text { c) conocer las nuevas tecnologías de información y comunicación y los distintos pro- } \\
\text { gramas que permiten su aplicación a la epidemiología. De este modo se aspira a crear } \\
\text { un aula virtual en la que la distancia física entre alumno y profesor ya no sea una barrera } \\
\text { a la enseñanza. Cada módulo tendrá un tutor y la instrucción se podrá impartir por co- } \\
\text { rreo postal, teléfono o correo electrónico. Además de la instrucción personalizada, habrá } \\
\text { una lista de discusión en Internet cuya finalidad será la de llevar a cabo discusiones en } \\
\text { grupo y resolver dudas en conjunto. Hay un número máximo de } 100 \text { plazas (mínimo de } \\
20 \text { plazas). Si la titulación del solicitante no es española, es necesario que le pida auto- } \\
\text { rización para participar en el curso al Vicerrectorado de Educación Permanente de la Uni- } \\
\text { versidad Nacional de Educación a Distancia (UNED). La fecha límite para la preinscrip- } \\
\text { ción es el } 15 \text { de octubre de } 2004 \text {. }\end{array}$ \\
\hline Información: \\
\hline $\begin{array}{c}\text { Trámites administrativos para personas sin títulos españoles: } \\
\text { Rosa Leocadio } \\
\text { Correo electrónico: rleocadio.cursos@fue.es } \\
\text { Contenido del curso: } \\
\text { Pablo Ruipérez García } \\
\text { Fundación Universidad-Empresa } \\
\text { c/ Serrano Jover } 5 \text { - 6 } \\
\text { 28015 Madrid, España } \\
\text { Teléfonos: (+34) } 915489868 \text { / } 915489860 \\
\text { Faxes: (+34) } 915417294 \text { / } 915470652 \\
\text { Internet: www.fue.es / www.same.ens.isciii.es }\end{array}$ \\
\hline
\end{tabular}

